
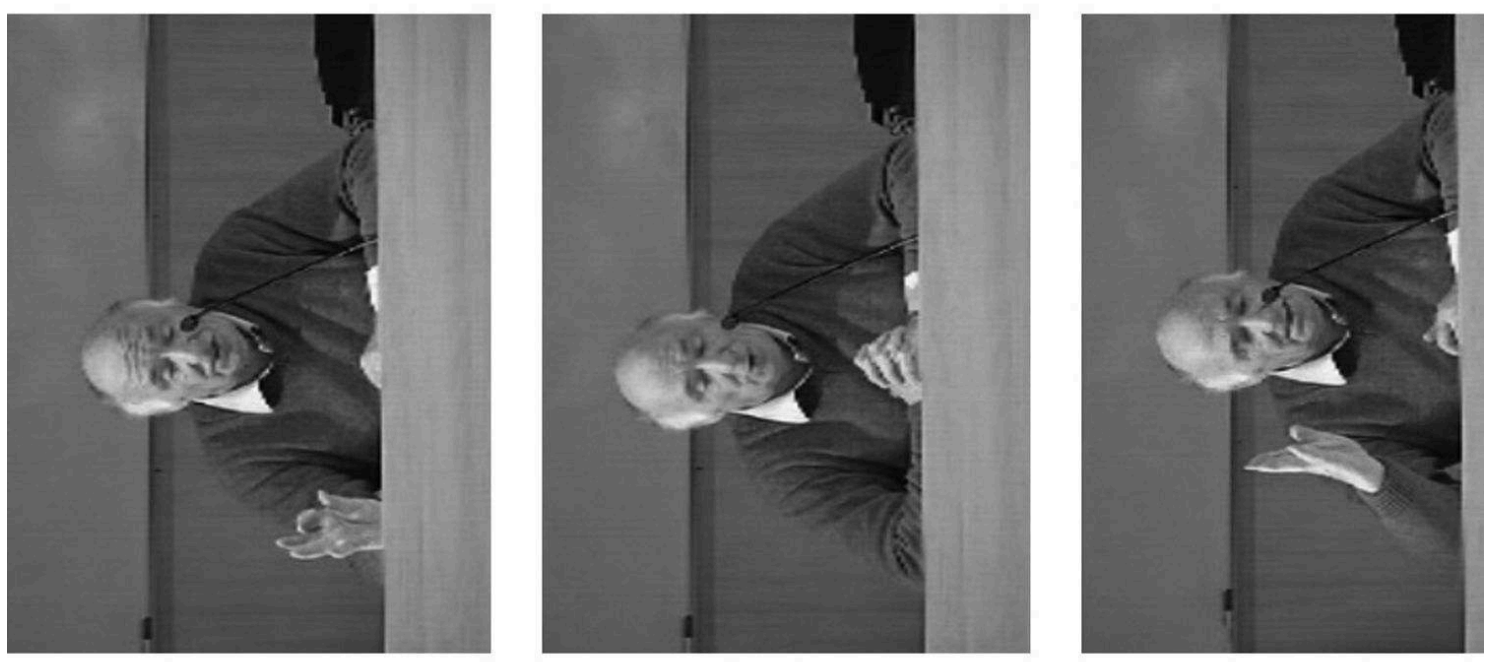

Jacques Roubaud

Lire, écrire ou comment je suis der 


\section{Lire, écrire ou comment je suis devenu collectionneur de bibliothèques}

Jacques Roubaud

DOI : 10.4000/books.pressesenssib. 111

Éditeur : Presses de l'enssib

Année d'édition : 2012

Date de mise en ligne : 20 juillet 2017

Collection : enssib2012

ISBN électronique : 9782375460252

\section{QboOKKs}

http://books.openedition.org

\section{Édition imprimée}

ISBN : 9782910227951

Nombre de pages : 48

\section{Référence électronique}

ROUBAUD, Jacques. Lire, écrire ou comment je suis devenu collectionneur de bibliothèques. Nouvelle édition [en ligne]. Villeurbanne : Presses de l'enssib, 2012 (généré le 01 février 2021). Disponible sur Internet : <http://books.openedition.org/pressesenssib/111>. ISBN : 9782375460252 . DOI : https:// doi.org/10.4000/books.pressesenssib.111.

(c) Presses de l'enssib, 2012

Conditions d'utilisation :

http://www.openedition.org/6540 

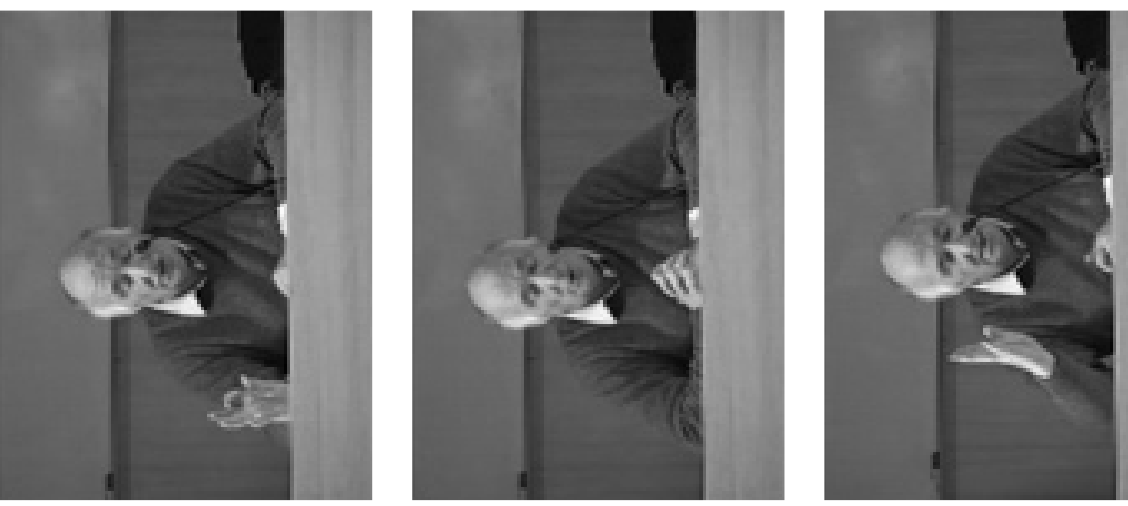

$\underline{\text { Jacques Roubaud }}$

\section{Lire, écrire ou comment je suis dev}


Presses de l'enssib

École nationale supérieure des sciences

de l'information et des bibliothèques

17-21 boulevard du 11 novembre 1918

69623 Villeurbanne cedex

Tél. 04724343 - Fax 0472444344

< http://www.enssib.fr/presses >

Lire, écrire ou comment je suis devenu collectionneur de bibliothèques [Texte imprimé]/Jacques Roubaud.

- Villeurbanne: Presses de l'enssib, cop. 2012. -

1 vol. (48 p.); $19 \mathrm{~cm}$. Version numérique disponible

gratuitement: < http://roubaudlireecrire.enssib.fr >

ISBN 978-2-910227-95-1 / $9 €$

Dewey: $028.8 \cdot 844$

Rameau

Bibliothèque nationale de France - Récits personnels - 1945-....

Bibliothèque de la Sorbonne (Paris) - Récits personnels - 1945-....

British library - Récits personnels - 1945-....

Roubaud, Jacques (1932-...) - Biographies 


\section{Lire, écrire ou comment je suis devenu collectionneur de bibliothèques \\ $\underline{\text { Jacques Roubaud }}$}


Un choix tout à fait personnel (et assumé)

parmi quelques œuvres de Jacques Roubaud

La Belle Hortense, Ramsay, Paris, 1985

Quelque chose noir, Gallimard, Paris, 1986

L'Enlèvement d'Hortense, Ramsay, Paris, 1987

L'Exil d'Hortense, Seghers (coll. Mots), Paris, 1990

Soleil du soleil, POL, 1990

Poésie, etc. etera, ménage, Stock, Paris, 1995

Le Grand Incendie de Londres, récit avec incises et bifurcations, Seuil, Paris, 1989 (Branche 1 du Projet)

Mathématique:, Seuil, Paris, 1997

(Branche 3, première partie, du Projet)

La forme d'une ville change plus vite, hélas,

que le cœur des humains. Cent cinquante poèmes.

1991-1998, Gallimard, Paris, 1999

Poésie:, Seuil, Paris, 2000 (Branche 4 du Projet)

La Bibliothèque de Warburg. Version mixte, Seuil (coll. Fiction \& Cie), Paris, 2002 (Branche 5 du Projet)

Graal théâtre: Joseph d'Arimathie - Merlin l'enchanteurGauvain et le Chevalier Vert - Perceval le Gallois Lancelot du Lac - L'enlèvement de la reine Morgane contre Guenièvre - Fin des Temps AventureuxGalaad ou la Quête - La tragédie du roi Arthur, avec Florence Delay, Gallimard, Paris, 2005 128 poèmes... composés en langue française, de Guillaume Apollinaire à 1968,

La Bibliothèque Gallimard, Paris, août 2006

Parc Sauvage, récit, Seuil (coll. Fiction \& Cie), Paris, 2008

Impératif catégorique, récit, Seuil (coll. Fiction \& Cie), Paris, 2008. (Branche 3, seconde partie, du Projet)

La dissolution, récit, Nous Eds, 2008

Le Grand Incendie de Londres, réédition des 5 premières branches du Projet en un seul volume, Seuil (coll. Fiction \& Cie), Paris, 2009 
Ce texte a été lu lors d'une conférence que Jacques Roubaud avait eu la gentillesse de donner, devant les élèves conservateurs de l'école nationale supérieure des sciences de l'information et des bibliothèques (enssib), en janvier 2008. Il était donc destiné à une forme orale, et le texte édité en conserve quelques traces.

Cette publication a été réalisée dans le cadre des 20 ans de l'enssib.

Thierry Ermakoff 


\section{Il a été dit que j'ai un rapport particulier aux bibliothèques,}

c'est pour cela que j'ai accepté tout de suite l'invitation qui m’a été faite. J'ai rédigé quelque chose que je vais vous lire.

Une, deux, trois fois par mois, depuis qu'à l'automne de 2001, ayant cessé d'être directeur d'études à l'EHESS, sigle qui désigne l'École des hautes études en sciences sociales, je suis devenu, non un retraité, mais, ce qui est plus noble, un pensionné de l'université inscrit sur le grand livre de la dette publique afin que la République française puisse me verser chaque mois la pension assez modeste à laquelle elle a décidé que j'avais droit en tant que vieux serviteur de l'Éducation nationale, une, deux, trois fois par mois, dis-je selon le cas, rarement plus, rarement moins, j'entre après avoir présenté aux agents de sécurité qui veillent à sa porte afin d'en interdire l'accès aux personnes principalement étudiantes ou non autorisées la carte que j'ai sortie de mon portefeuille dès ma descente de l'autobus 
revêtue d'une pastille ronde et jaune qui atteste que, pendant l'année universitaire 2007-2008, j'ai le droit de franchir ce barrage dans la cour de la Sorbonne à Paris.

Ça, c'est mon introduction, elle fait 1000 caractères exactement en une seule phrase.

C'est une contrainte très intéressante.

\section{Je traverse la cour en} oblique, monte un escalier qui me conduit à la bibliothèque où je pénètre

après avoir sonné par l'entrée réservée aux professeurs, montre ma carte marquée verticalement en rouge "magasin" et me dirige vers le service du prêt. Là, je sors de mon cabas un carnet semblable à celui dont j'ai extrait les feuilles où mon imprimante Canon a inscrit le texte que j'ai composé pour mon bavardage d'aujourd'hui devant vous, et je restitue les livres que j'ai emprunté une, deux, trois ou quatre semaines auparavant selon le cas. J'ai le droit de les conserver un mois, 
pas plus. Je prends bien soin de vérifier que ma restitution est exactement enregistrée sur l'écran réservé à l'enregistrement des prêts, car je n'ai aucune confiance en l'appareil qui tient ces données en mémoire, et je crains, si je ne m'assure pas que tout va bien, d'être considéré comme ayant encore en ma possession tel et tel livre par suite de la mauvaise volonté du logiciel de l'ordinateur, alors que je sais que je l'ai ramené lors de ma dernière visite. Une telle aventure m'est déjà arrivée et m'a mis au bord de la crise cardiaque. Je me voyais déjà exclu du prêt, menacé de je ne sais quelle terrible sanction. Heureusement, une bibliothécaire compatissante persuada le magasinier d'aller aussitôt vérifier que le livre en question avait déjà rejoint sa place attitrée et je pus, battements de mon vieux cœur apaisés, considérer calmement de nouveaux emprunts. Depuis cette aventure, je ne pars pas à la recherche des cotes que j'ai notées sur mon carnet avant d'être sûr, absolument sûr que tout est en ordre. Mais pourquoi, me direz-vous, et vous seriez en droit de me le dire, pourquoi, alors que vous pourriez jouir d'un repos bien gagné, qu'aucune obligation professionnelle de préparation de cours par exemple 
ne vous y oblige, pourquoi continuez-vous avec tant de constance à vous rendre pour y emprunter un, deux, trois, quatre, cinq, même six livres (maximum autorisé), une, deux ou trois fois par mois à la bibliothèque de la Sorbonne? Et pourquoi d'ailleurs dans cette bibliothèque-là précisément, alors que, quand vous étiez en activité (professionnellement un mathématicien), et que depuis bien longtemps cette bibliothèque a cessé d'être une bibliothèque universaliste, et que d'autres bibliothèques que vous connaissez certainement comme celle de l'IHP (Institut Raymond Poincaré) offrent un bien plus grand choix d'ouvrages dans la discipline mathématique? Je vais m'efforcer de répondre à ces questions. Je sais que vous ne les avez pas posées, et pour cause, mais j'espère que vous voudrez bien me pardonner l'emploi de ces antiques procédés rhétoriques. Je traiterai d'abord de la deuxième question.

Je ne me suis pas, je l'avoue, dans ma vie, uniquement occupé de mathématiques, et je suis d'ailleurs aujourd'hui un retraité de la mathématique mais j'étais aussi depuis ma jeunesse, mon enfance même, et continue d'être, un poète, un compositeur de poésies, activité que je ne considère pas à la 
différence de la plupart des lecteurs, libraires et journalistes comme obsolète. J'ai sans doute tort. La façon dont va le monde tend à me montrer de plus en plus que j'ai tort mais c'est ainsi. Il y a une bonne douzaine d'années, en 1995 exactement, j'ai reçu un jour une proposition qui m'a surpris. On me demandait de participer à une nouvelle traduction de la Bible. Il n'existe pas, me dit-on, de traduction française de la Bible qui fasse autorité. Les Anglais ont la Bible du Roi James, que je connaissais depuis l'enfance, car ma mère était professeur en anglais, ce livre était en bonne place dans la bibliothèque de mes parents à côté de Shakespeare. On ne peut pas raisonnablement enseigner la littérature anglaise sans connaître ces deux œuvres, la Bible du Roi James et Shakespeare. Les Allemands eux, ont la Bible de Luther, mais rien de tel en langue française. Il y a eu depuis la Renaissance plusieurs versions, aucune ne s'est imposée à tous et j'appris ainsi qu'il existait le projet d'une traduction nouvelle qui serait une œuvre collective. Et son originalité serait la suivante: chaque livre, dans ce que les chrétiens nomment Ancien et Nouveau testament, serait traduit par des écrivains associés à des biblistes. 
Il y a de très bonnes traductions sur le marché, par exemple la Bible de Jérusalem mais aucune, selon les initiateurs de ce projet, ne tient vraiment compte du fait que la Bible est aussi un grand texte littéraire.

Bien, et pourquoi faisait-on appel à moi? On me dit très simplement qu'il y avait un livre dont le titre français est Nombres. Alors, comme je suis mathématicien, j'étais tout indiqué. J'objectai que je n'étais pas croyant, que j'étais un agnostique. Je précise un agnostique, pas athée. On me répondit que c'était sans importance. Parmi les écrivains qui allaient participer, il y avait des catholiques, des protestants, etc. L'éditeur de cette Bible serait la maison des Éditions Bayard. Après quelques hésitations, j'acceptai mais je posai une condition. D'être aussi associé à la traduction du livre de Qohelet, l'Ecclésiaste pour la traduction française. On me l'accorda. Et bien sûr, comme il est impossible de comprendre le livre Nombres sans le livre intitulé Lévitique, j'ai dû aussi travailler sur ce livre là et, bref, ça m’a occupé plusieurs années jusqu'à la publication de la Bible Bayard, un peu, juste un peu, deux ou trois jours juste après le 11 septembre 2001. Pendant les six 
années où j'ai participé à cette entreprise passionnante de traduction, j'ai eu la curiosité de me renseigner sur les traductions françaises et autres du texte biblique. J'ai fréquenté bien sûr la Bibliothèque Nationale mais j'ai eu aussi recours à la bibliothèque de la Sorbonne. Mes lectures m'ont mis sur la trace d'une traduction restée à peu près inconnue depuis le milieu du XVI e siècle, celle de Sébastien Castellion ou Chateillon. Elle m'a passionné pour sa beauté, son originalité et par la personnalité de son auteur qui est proprement extraordinaire. Castellion, en effet, restera dans l'histoire, on s'en rend compte de plus en plus, et les études sur lui aujourd'hui se multiplient, comme le premier homme du XVI e siècle à avoir conçu puis défendu l'idée de tolérance religieuse et ceci au beau milieu des heurts terribles entre protestants et catholiques. D'abord converti à la Réforme, disciple et ami de Calvin, il rompit avec lui à l'occasion de l'affaire Servet, cet hérétique que les calvinistes brulèrent, reproduisant ainsi le triste exemple de l'Inquisition. Il fut le seul homme en Europe à protester contre ce supplice et écrivit cette phrase admirable qui mérite d'être méditée encore aujourd'hui : tuer un homme, 
ce n'est pas défendre une doctrine, c'est tuer un homme (écrit en 1560). Il existe une grande biographie de Castellion, qui date de la fin du XIX', par Ferdinand Buisson. Et je l'ai empruntée plusieurs fois à la bibliothèque de la Sorbonne pendant les années de ce travail. Avec la publication de la Bible Bayard, avec mon retrait de la vie active, j'aurais pu cesser de me préoccuper de ces questions. En fait, j'ai continué à suivre les recherches sur l'histoire du texte biblique depuis ses origines. De très nombreux articles et ouvrages, paraissent chaque année sur ces questions et le domaine est en plein bouleversement. Or, mes moyens ne me permettent pas de faire l'acquisition de tous ces gros livres publiés aux États-Unis, en Angleterre, aux Pays-Bas... et j'ai donc conservé l'habitude de me rendre à la bibliothèque de la Sorbonne et d'y emprunter les livres sur ce sujet. Bien sûr, il n'y a pas tout, il faut aussi aller à la bibliothèque de France, et à la bibliothèque de France il n'y a pas tout non plus, il y a des choses que je voudrais lire et j'ai été obligé ainsi de me rendre deux ou trois fois à Londres pour des séjours à la British Library que je fréquente aussi depuis plus de trente ans. Mais, avec la bibliothèque de la Sorbonne, j'ai de 
quoi nourrir mes lectures et profiter surtout de la présence des volumes dans ma propre bibliothèque pendant un temps raisonnable. La raison principale qui m'a fait continuer à choisir ce mode de consultation est la suivante: je possède un privilège inouï, je peux aller prendre des livres dans les magasins. Vous n'imaginez pas ce que c'est. Je bénéficie de ce droit depuis trente-sept ans. Depuis qu'en 1970 j'ai été nommé professeur de mathématiques à l'université de Paris X Nanterre, je l'ai conservé comme directeur d'études à l'EHESS et je ne l'ai pas perdu depuis. Mais je sais que c'est vraisemblablement la dernière année où je pourrai profiter de cet avantage. La bibliothèque de la Sorbonne va, m'a-t-on dit, fermer cet été pour réfection et sans doute déplacement, ceci pour deux années au moins, et je sais que même si je suis encore en vie, en bonne condition physique et intellectuelle, au moment de sa réouverture, il est clair qu'un tel privilège ne sera pas renouvelé. Cela donne à mes visites en ce lieu aujourd'hui, un caractère d'urgence et de nostalgie anticipé particulièrement intense. Un bouton est pressé, la porte d'accès au magasin s'ouvre, je la franchis. Dans ma poche, j'ai le carnet où j'ai 
noté un certain nombre de cotes et me voici dans le magasin A. Il y a deux magasins, magasin A et magasin B. Je vérifie l'emplacement des cotes des livres qui m'intéressent, je les connais en fait assez bien mais l'accumulation incessante de livres nouveaux fait qu'il faut sans cesse effectuer des déplacements de cotes entières, et je ne peux pas entièrement me fier entièrement à mes souvenirs. Je vais au fond du couloir vers les ascenseurs que les professeurs sont priés de prendre pour ne pas gêner les magasiniers qui ont le leur. Selon le cas, je monte vers les étages ou bien je descends dans les sous-sols. C'est une véritable aventure. Il y a en effet deux types de sous-sols, les sous-sols en quelque sorte ordinaires et les sous-sols dits Turgot. Or, ils ne sont pas exactement au même niveau, leurs accès diffèrent. Dans certains cas, il me faudra descendre quelques marches, dans d'autres en monter quelques autres, et il m'arrive encore de me tromper et de me retrouver deux fois de suite devant la cote L au lieu de la cote $\mathrm{M}$, par exemple, car je n'ai guère le sens de l'orientation. Les portes qui s'ouvrent dans les escaliers entre des étages sont généralement dépourvues d'indications sur la nature des 
cotes qui se trouvent derrière elles. J'erre ainsi souvent entre les deuxième et troisième sous-sols sans me reconnaître. Parfois, je tombe par hasard sur le bon endroit et il me faut ensuite retrouver la bonne sortie pour rejoindre le bon ascenseur. J'essaie par orgueil quand je suis perdu de ne pas demander mon chemin à un magasinier que je croise pour ne pas affronter un regard qui soit, soit excédé, soit, ce qui est pire, aimable, bienveillant mais ironique. Je pourrais vous guider dans ce rayon mais cela prendrait trop de temps. Je ne dirai quelques mots que de mon magasin favori, le magasin B. Pour atteindre le magasin B, il faut, puisque on est entré par le magasin $A$, grimper deux étages, monter quelques marches, franchir une passerelle, redescendre quelques marches. Un peu de jour, pas beaucoup, pénètre par les fenêtres qui ont été lavées pour la dernière fois en 1911. Les cotes les plus élevées, celles des vieux volumes de théologie par exemple, sont au huitième. Les escaliers aux marches de bois sont étroits, leurs marches craquent, les rampes tremblent, brinqueballent. Si les escaliers vous inquiètent, vos jambes mal assurées de septuagénaire vous forcent à prendre l'ascenseur. C'est 
un monte-charge infiniment lent qui vous inquiète encore plus peut-être, tant sa réticence se fait entendre par les grincements métalliques inquiétants: seule l'indécision est manifeste. On croise très peu d'êtres humains dans ces hauteurs; plus on monte, plus on est seul. Les plafonds sont bas, les rangées de livres sont obscures. Tout au fond d'une rangée, le livre que vous cherchez sans doute est au ras du sol. On s'agenouille, cherchant à déchiffrer la cote et le titre, et on se dit alors avec angoisse que peut-être on ne parviendra pas à se relever. On appellera, on appellera encore, votre voix faible ne parviendra à aucune oreille, les heures passeront, ce sera la fermeture, ce sera la nuit. J'imagine parfois que, dans un recoin particulièrement obscur, le cadavre desséché de quelqu'un de mes prédécesseurs attend encore après quarante ou cinquante ans d'être découvert. Je ne manque jamais quand je viens ainsi chercher quelques livres à emprunter dans les magasins de la bibliothèque de la Sorbonne de penser à Aby Warburg. Vous connaissez sans doute son principe du bon voisin qui a été rapporté par Gombrich dans sa biographie du grand historien d'art. On peut l'exprimer comme ceci: dans une bibliothèque 
bien faite, le livre dont vous avez vraiment besoin, se trouve à côté de celui que vous êtes venu chercher. Telle était faite la propre bibliothèque de Warburg. Cette œuvre d'art, qui a été transportée en Angleterre par les disciples du Maitre, à la barbe des nazis peu après la venue au pouvoir d'Hitler, se trouve maintenant hébergée par l'université de Londres. Certes, la bibliothèque de la Sorbonne ne peut pas réellement satisfaire au PDB, Principe du Bon Voisin, mais je ne manque jamais quand je prends un livre dans un de ces rayonnages de regarder ceux qui se trouvent à proximité. J'ai fait ainsi des découvertes inattendues et heureuses. C'est là un bénéfice supplémentaire de mon privilège, car je ne peux pas agir ainsi quand je suis à la Bibliothèque Nationale, je veux dire quand je suis aujourd'hui à la bibliothèque François Mitterrand ou bibliothèque de France mais à laquelle je continue à penser sous le nom ancien de Bibliothèque Nationale. 
Je vous lis comme interlude, un sonnet que j'ai composé en 1993 et qui s'appelle Square Louvois. C'est là que se trouvait jadis la Bibliothèque Nationale.

\section{Square Louvois}

Peut-être mille fois en trente ans, je me suis assis Sur un banc vert dans le square Louvois.

Le soleil sur les yeux, seul, entouré des bruits De fontaines bavant et traversé de voix.

À l'éblouissement des jets d'eaux j'étais, moi, Possesseur du trésor de livres inouïs Potentiellement convocables depuis Les magasins de l'ex-bibliothèque du Roi.

Saône et Seine et Garonne et Loire, je voyais Les quatre nymphes fleuves aux regards noyés. Je contemplais leurs seins de bronze allégorique

Puis je retraversais la rue de Richelieu Pour rejoindre ma place et, lecteur euphorique, Jouir en souverain d'un républicain lieu.

Voilà, un hommage à la Bibliothèque Nationale. 


\section{J'ai été introduit, disons mieux, présenté à la Bibliothèque Nationale par mon premier beau-père,}

Paul Benichou, en 1963. Il était alors professeur à Harvard et, quand il revenait à Paris pour les vacances, il poursuivait les recherches d'histoires littéraires qui devaient aboutir aux quatre volumes récemment repris aux Éditions Gallimard sous le titre Romantisme français. Il occupait toujours la même place, la place 115, dans la grande salle de lecture. Sa fille Sylvia, jeune agrégée d'espagnol, avait commencé une thèse et tout naturellement y venait aussi étudier. La première fois qu'elle avait franchi les portes augustes de la salle Labrouste, son père l'avait présentée au chef des magasiniers avec lequel il entretenait d'excellentes relations. Chaque nouvelle année, il lui confiait l'enveloppe contenant les étrennes qu'il destinait à tous ceux qui apportaient les livres aux lecteurs à leur place et le chargeait de les distribuer. Cet excellent homme n'avait pu s'empêcher de s'exclamer en voyant Sylvia, à l'intention de Paul Bénichou: «Comment, si jeune, et vous la mettez déjà dans les livres! » À cette époque, j'étais en train, outre 
mon enseignement à la faculté des sciences de Rennes, de mener de front deux entreprises. Des recherches en vue d'une thèse de mathématique d'une part, d'autre part la composition d'un livre de poèmes pour lequel j'avais choisi de rendre hommage à une forme poétique aussi anti-surréaliste que possible, la forme sonnet. Je lisais, à cet effet, tous les sonnets qui me tombaient sous l'œil, anciens et nouveaux, aussi bien en italien, anglais, espagnol ou portugais. Paul Bénichou me fit observer qu'il y avait un moyen moins ruineux que la fréquentation des libraires, (il pensait au biberon de sa petite fille et aux commandes à l'étranger) pour avancer dans mes investigations. Et c'est ainsi que j'entrai à mon tour dans ce temple du livre qu'Alain Resnais a célébré en 1956 dans son beau film, Toute la mémoire du monde. Je n'ai pas quitté cette salle sans regret. Je ne vais pas ici parler de la nouvelle et très grande bibliothèque à laquelle, non sans mal, j'ai fini par m'habituer une fois oubliés certains épisodes assez burlesques de ses deux premières années. Mais gardons le silence. Je suis sensible à ses défauts et je me place, bien sûr, d'un point de vue exclusif de lecteur, mais j'apprécie par exemple 
la possibilité qui est aujourd'hui la mienne de réserver d'avance une place à partir de mon bureau par quelques manipulations simples sur mon ordinateur et d'obtenir ainsi quantité raisonnable d'ouvrages. Dans les derniers temps de son existence, avant le transfert de ces livres vers le nouveau bâtiment du bord de Seine, la bibliothèque étouffait et la vie des lecteurs dans la salle de lecture était devenue très difficile. En 1985, j'ai publié une sorte de parodie de roman, le premier d'une série prévue de six romans dont trois ont été publiés; le titre est La belle Hortense. Et dans un style un peu burlesque, j'ai transformé cette expérience de lecteur dans les derniers temps de la Bibliothèque Nationale en fiction. Je précise bien que c'est une fiction. Je vais vous lire un passage. «Une fois franchit le sas d'entrée, protégé défendu comme l'accès à une capsule d'astronautes, grâce à la présentation de sa carte munie d'un rectangle orange en plastique épais et translucide indiquant le numéro de sa place, c'était sa place habituelle, ayant déposé le tout carte et rectangle à un comptoir où LA bibliothèque les gardait en otages, (un peu, comme si l'on en croit les films de gangsters, on abandonne son identité et ses effets personnels 
au moment d'entrer en prison), Hortense, c'est l'héroïne, jetant son sac et ses cahiers, sans oublier l'en-cas de $\mathrm{M}^{\text {me }}$ Groichant, qu'elle avait pu emmener sur sa table, se précipita vers la salle des catalogues afin de repérer au plus vite les cotes des ouvrages qu'elle rêvait d'obtenir. La stratégie défensive de la bibliothèque, en effet, obligée par la loi et la coutume de permettre au lecteur autorisé par la possession d'une carte (obtenue non sans mal après une longue enquête de sécurité et le remplissement de l'insidieux questionnaire qui permettait d'en éliminer plus d'un) qui permettait la consultation des ouvrages qui lui appartiennent en propre, qui sont sa gloire, son douaire et son trésor, et qu'elle ne cesse de caresser, de contempler, d'adorer dans le silence sombre de ses magasins, consistait à retarder le plus possible le moment où elle aurait à les sortir et à les soumettre aux regards salissants de ces ignares, dont elle soupçonnait d'ailleurs que l'intention secrète était de les barbouiller, de les lacérer, de les griffonner, de les détériorer ou tout simplement de les voler. Il s'agissait pour elle d'atteindre le moment béni de l'après-midi où une cloche (tocsin pour les lecteurs, carillon de liesse 
pour elle) annonçait la fin des communications d'ouvrages pour la journée en ayant livré le moins possible de livres à la convoitise des barbares. C'est pourquoi, dès qu'il avait réussi à pénétrer dans la forteresse, le lecteur devait agir avec la plus grande célérité et prestesse et c'était la raison de la bousculade effrénée dans les escaliers conduisant la salle des catalogues à laquelle Hortense pris part ce matin-là en excellente position.

La difficulté première consistait à découvrir la cote de l'ouvrage, soigneusement dissimulée. Il n'y avait pas en effet, comme on aurait pu s'y attendre, par exemple, une suite de volumes indiquant, pour chaque auteur selon sa place alphabétique, les ouvrages disponibles, non; si Hortense avait envie de lire Pierrot mon ami de Raymond Queneau par exemple, elle devait savoir à quel moment le livre avait été acquis, pas celui (ça aurait été trop simple) de la parution. Il y avait pour chaque tranche alphabétique, de manière parfaitement indécidable, un volume valable pour certaines de ces années seulement, situé dans un endroit totalement imprévisible de la salle. Il fallait le repérer, chercher l'auteur, chercher l'ouvrage, noter la cote et ensuite déterminer dans 
quel autre volume se trouvait maintenant la cote réelle, car la cote première était une cote ancienne qui avait été abandonnée au profit d'une autre, plus moderne, lors d'un quelconque changement de règne à l'intérieur de l'empire bibliothécaire. Il va de soi que seule une très longue habitude, ou l'héritage d'une tradition secrète, ou l'amitié d'un bibliothécaire, pouvait permettre de s'y reconnaître. Plus d'une fois déjà, Hortense avait dû consoler quelques malheureuses étudiantes américaines à peine sorties des plages rassurantes de la bibliothèque du Congrès à Washington, sanglotant dans une douzaine de Kleenex aux pieds obscurs de quelques rayons.

Mais ce n'était pas tout! Admettons que vous ayez réussi, par miracle, à trouver la cote du livre que vous cherchiez, ou que tout simplement renonçant à la déterminer, vous ayez en désespoir de cause pris la première qui vous tombait sous la main; que vous ayez correctement, disons, rempli les bulletins de demande de chaque livre et déposé ceux-ci dans la boîte réservée à cet effet, vous n'étiez pas au bout de vos peines, et la Bibliothèque, bien qu'ayant perdu la première escarmouche, n'était pas vaincue pour autant. Car alors 
commençait une longue attente, pendant laquelle, pensiez-vous naïvement, on s'affairait, toutes affaires cessantes, à la recherche de vos ouvrages, afin de vous les apporter. Vous attendiez, une demi-heure passait, une heure, rien. Vous aviez terminé votre courrier, levé plusieurs fois les yeux vers l'immense coupole vitrée, à travers la poussière de laquelle filtrait un peu de jour, et voilà qu'un des livreurs de livres se présentait devant la rangée où vous étiez assis. Et voilà qu'il jetait sur votre table un livre! Vous le preniez fébrilement: hélas! ce n'était pas Pierrot mon ami de Raymond Queneau, dont vous aviez, grâce à un tuyau sûr, déterminé la cote dans un souscatalogue spécial consacré aux ouvrages sur le cirque, que vous aviez devant vous, mais Einfürhung in der Theorie des Elektrizität und der Magnetismus de Max Planck, Éditions de Heidelberg, 1903. Vous vous précipitez au bureau des réclamations. Vous attendez dix minutes. Une Finlandaise ne comprend pas pourquoi La revue critique du discobole français, année 1910, ne se trouve pas dans cette salle alors que l'année 1909 y est. La bibliothécaire explique patiemment dans un allemand approximatif que le conservateur a 
décidé de transférer, pour des raisons de sécurité, toutes les revues sportives à partir de 1910 précisément dans une autre salle, qui d'ailleurs vient de fermer. Enfin, c'est votre tour. La confrontation de votre bulletin de demande et de la cote du livre de Planck montre clairement que vous avez raison. Le $Z$ n'est pas un $W$, le 8 n'est pas un 4 . Il n'y a aucun doute, mais que faire? Attendre encore une heure? Le livre qui arrivera s'il n'est pas Pierrot mon ami, sera peut-être encore moins intéressant. Résigné, vous regagnez votre place et vous commencez l'étude de théorie des quanta. La première stratégie, donc, était la stratégie de l'erreur, dont une variante était l'envoi du bon ouvrage à un autre lecteur. On voyait ainsi dans l'allée centrale de la salle de lecture les chercheurs fébriles essayant d'échanger, en des échanges souvent triangulaires, un ouvrage sur la cuisine pygmée contre l'édition originale des Prolegomena rythmorum du père Risolnus. Mais il y avait un échange supérieur dans la dissuasion: c'était l'emploi d'une arme particulièrement redoutable, la panoplie des réponses dilatoires que les magasins envoyaient au lecteur par l'intermédiaire de son propre bulletin de demande; Ces réponses 
pouvaient prolonger la lutte pendant plusieurs journées; si cette stratégie était choisie, cela se passait de la manière suivante: le distributeur de livres apparaissait dans votre rangée avec son chariot; il n'y avait rien pour vous; une demiheure supplémentaire passait. Vous receviez alors votre bulletin de commande, généralement chiffonné, portant l'indication: "manque en place». Le lendemain, vous redemandiez l'ouvrage; la réponse était cette fois: "cote à revoir ". Le troisième jour c'était «à la reliure » et enfin le quatrième, par un raffinement de cruauté dont on appréciera toute la saveur: "communiqué à vous-même le... » et suivait alors la date de votre première demande. C'était le degré ultime de l'escalade, car vous vous trouviez alors dans un état inconfortable, tentant d'expliquer que vous n'aviez jamais eu communication de l'ouvrage, avec le sentiment pénible qu'on vous prenait pour un imbécile, un distrait ou un voleur. Les bibliothécaires essayaient de vous consoler et vous lisiez dans leur regard apitoyé le jugement sans appel: le malheureux, elle a encore frappé ! Il va s'en dire que vous appreniez, si vous ne vous découragiez pas définitivement et ne preniez pas 
immédiatement l'avion pour Londres afin de vous consoler au British Museum, vous appreniez, à l'usage, à déjouer certains de ces pièges. Contre la tactique de la réponse dilatoire, par exemple, la contre-attaque consistait en un renoncement instantané au profit d'un autre ouvrage, et un nouveau sondage pour le livre que vous désiriez initialement, plusieurs jours plus tard, ce qui obligeait l'ennemi à des efforts considérables de mémoire qu'il ne tardait pas à trouver très onéreux. Aussi, pour des lecteurs un tant soit peu aguerris, les procédés de dissuasion courante, dont nous venons de donner quelques échantillons étaientils insuffisamment efficaces. C'est pourquoi la Bibliothèque inventait sans cesse de nouvelles stratégies: alerte à l'incendie, retard de l'horloge dans le hall d'entrée permettant de gagner une bonne demi-heure à l'ouverture des portes (l’heure véritable était rétablie dans la journée, avancée même, ce qui permettait un gain à la sortie). La dernière en date, qui avait désarçonné même Hortense et envoyé un membre de l'Institut à l'hôpital avec une crise nerveuse, consistait à fermer brusquement, pour une durée indéterminée et sans préavis, un magasin entier. Ainsi 
le lundi, on ne communiquait pas la poésie, le mardi pas de mathématique; pas de livres d'histoire de la navigation, ni postérieur à 1863, le mercredi. Cette offensive, récente, semblait couronner de succès, et le découragement s'emparait de certains des plus tenaces des lecteurs. On vit un spécialiste fameux de la rhétorique à la Renaissance convoquer la presse, et entouré de sa femme et de ses quatre enfants en larmes, annoncer qu'il renonçait et entrait dans l'immobilier. De nombreux lecteurs, naïfs, crurent qu'en s'adressant à quelque pouvoir, on pourrait modifier le cours des choses; ils formèrent un comité de lecteurs, lancèrent une pétition, interpellèrent à la Chambre des députés et au Sénat, agirent sur les amicales d'anciens élèves des Grandes Écoles. La Bibliothèque sourit dans sa barbe. Il y eu des élections pour une assemblée représentative des usagers, avec scrutin de listes à deux tours majoritaires semi-proportionnel, avec panachage; une boite de réclamation fut apposée à l'entrée à l'usage des lecteurs, le chauffage fut amélioré dans le département des manuscrits sportifs, des carrières politiques s'ébauchèrent et se fut tout. » 


\section{J'ai passé aussi beaucoup de temps dans une salle de lecture d'une autre grande bibliothèque.}

Moins qu'à la Bibliothèque Nationale mais beaucoup d'heures quand même. C'est celle de la British Library à Londres. Mais elle aussi a fini par succomber. Et j'ai noté le moment de ma dernière visite à cette bibliothèque, juste avant qu'elle ne ferme pour ensuite ouvrir dans une autre partie de Londres, plus loin. À partir du 24 novembre de la présente année 1997, ai-je écrit à ce moment-là, la salle de lecture de Panizzi (c'est lui qui avait inventé cette salle, lieu parfait et paradisiaque de la lecture publique) sera dérobée aux individus de mon espèce qui seront exilés du coté de SaintPancras. Dans une nouvelle bibliothèque, Nouvelle Library que le prince de Galles compara un jour d'énervement, en tant qu'objet architectural, à un Wimpy. Opinion qu'après usage, six ans plus tard, je ne partage pas du tout. Le prince de Galles, j'ai le regret de l'écrire est à mes yeux un imbécile prétentieux et sa «Camomille» de maîtresse ne vaut 
guère mieux. Ayant pris connaissance, le 12 mars, de la notice qui informait les lecteurs de cette fatale nouvelle, assis à la place qui fut la mienne dans cette salle de très nombreuses journées, pendant de très nombreuses années, et certainement parmi les journées les plus heureuses de ma vie, ou peut-être parmi ses journées heureuses les heures les plus heureuses, furent celles que j'y passais les soirs d'été aux ouvertures plus tardives, jusqu'à neuf heures du soir passées, ou je tentais de profiter le plus possible d'une durée pour moi luxueuse, réservant le plus pour ces moments les lectures qui devaient être faites dans la North Library, l'équivalent Londonien de la réserve de la BN. J'étais à la place R14. Celle que je prenais toujours quand je venais à Londres, et à l'instant de me lever pour sortir, je me suis dit: «Et voilà, c'est fini. Je n'y reviendrai plus jamais. » Il n'y avait aucun corbeau sous le dôme bleu et pourtant j'ai entendu distinctement un «never more» des plus lugubres. Je n'ai pas voulu laisser cet instant sans trace, j'ai rouvert mon cahier et j'ai écrit: «British Library, 12 mars 1997, good bye. » M'étant assis à ma place pour cet acte de mise en mémoire, je n'arrivais plus à partir, il me semblait que cet 
adieu n'était pas suffisant à la circonstance, et regardant autour de moi, dessus, dessous, à droite, à gauche, pour essayer d'emmagasiner dans mon peu fiable souvenir le plus possible d'images de ces lieux, que j'avais tant de fois vus comme quelqu'un qui pense devoir les revoir encore et pour ainsi dire toujours, j'ai aperçu en face de moi la petite note dactylographiée vénérable, vétuste, caduque depuis le début de la détérioration, ensuite sans cesse accélérée, du fonctionnement de la bibliothèque dès lors que son départ définitif a été annoncé et je l'ai copiée dans mon cahier. (La détérioration de l'état de la British Library au moment où comme la Bibliothèque Nationale elle n'arrivait plus à fonctionner, car il y avait trop de lecteurs, était affrontée de manière différente de celle que j'ai décrite plus haut, avec quelque exagération, pour sa sœur parisienne: tout le monde y est resté toujours très aimable mais les livres n'arrivaient pas. Tout simplement pas. On attendait un jour, deux jours, trois jours...) J'ai copié cette description: la salle de lecture devant être envahie par le musée et son intérieur, sans aucun doute saccagée et changée au point de ne plus être reconnaissable. Il est fort possible que les détails que je consigne 
ici soient effacés de la mémoire bibliothécaire, et que mon écrit en conserve, après la mort de tous les lecteurs qui lirent là, et qui en préservèrent dans leur mémoire faillible et provisoire l'image, la seule trace. Et j'ai ajouté en dessous des détails concernant ma place, la place R14. Bleue, place bleue, du même bleu que le plafond. C'est plutôt d'ailleurs une couleur que j'aimerais, le bleu Panizzi, celui qui a inventé cette salle, contenant un peu de vert, un peu de gris, présente partout. Une couleur apaisante, idéale pour la lecture méditative, intense mais pondérée, rendue encore plus noble et adéquate par la patine de toutes ces années de pensées de lecteurs qui s'élevèrent, et se répandirent dans l'atmosphère sereine de la Reading Room. J'ai souvent pensé que ma place R14 pouvait être proche de celle qu'occupait Karl Marx quand il allait à la British Library, et bien d'autres. Quelqu'un a créé ce lieu qui était vraiment un lieu enchanteur. Panizzi, qui conçu ce lieu, était un carbonaro, un conspirateur de Florence au XIX siècle. Il avait conspiré, en carbonaro qui se respecte, dans une conspiration dont le but était d'assassiner le Grand Duc de Florence et d'instaurer illico la République et le bonheur sur les terres 
des princes de Médicis. Comme on aurait pu s'en douter, la moitié des conspirateurs étaient des agents du ministre de la police du Duc. Panizzi put échapper à l'arrestation, se réfugia en Angleterre, où il vécut quelque temps en donnant des leçons d'italien. Il raconte dans ses mémoires qu'il reçut un jour, dans son refuge de la libre Angleterre, une lettre très officielle du ministre du Duc, qui lui réclamait, accompagné d'une facture détaillée, le remboursement de la somme que le grand-duché aurait dû dépenser pour son exécution qui n'avait pu avoir lieu, en raison de son absence injustifiée à cette cérémonie. C'est vraiment extraordinaire. Préparant ma venue devant vous, je me suis employé à recenser les bibliothèques que j'ai fréquentées plus ou moins longuement au cours de ma vie depuis mes années d'étudiant. Elles sont très nombreuses. J'avais commencé à en faire une liste puis je me suis arrêté.

À Paris, la Bibliothèque Nationale, la bibliothèque Sainte-Geneviève, la bibliothèque de l'Arsenal, la bibliothèque d'histoire du protestantisme français, la bibliothèque historique de la Ville de Paris, la bibliothèque de l'Institut Henri Poincaré pour la mathématique. À Londres, la British Library. 
À Oxford, la Bodleian. À Washington, la Library of Congress (la bibliothèque du Congrès), la New York Public Library, une énorme bibliothèque, magnifique, etc., etc. Il manque à mon tableau de chasse, et j'en ai quand même beaucoup, et c'est un très grand regret pour moi, la bibliothèque de Wolfenbüttel, qui eut au XVIII siècle pour directeur Leibniz, vous vous rendez compte, c'est très impressionnant. Ce Grand Duc de Wolfenbüttel a créé cette bibliothèque à la fin du XVIe siècle, en 1572, et c'était un amateur de livres; pas pour les lire, seulement pour les avoir. Donc, il avait envoyé dans toute l'Europe des agents spéciaux qui achetaient tout ce qu'ils trouvaient, et ils ramenaient leur bution à Wolfenbüttel. Ce qui fait qu'il y a dans cette bibliothèque des tas de livres qu'on ne trouve pas ailleurs, parce qu'ailleurs ils ont disparu. Une merveilleuse bibliothèque, mais je n'ai jamais eu l'occasion d'y aller.

Une famille très spéciale de bibliothèques doit être considérée à part. Dans les années soixante-dix et quatre-vingt, je me suis mis en tête d'explorer assez systématiquement, la forme du sonnet de langue française pendant le premier siècle de son existence. De Marot, 1530, à Malherbe 1630. 
L'aboutissement de ces recherches a été d'une part une seconde thèse (seconde, parce que j'avais fait une thèse de mathématiques), une seconde thèse de littérature française; d'autre part, une anthologie qui se nomme Soleil $d u$ soleil et que l'on trouve en «Poésie poche» chez Gallimard aujourd'hui. Il s'y trouve certains des sonnets que j'ai lu dans ces endroits-là. Beaucoup n'avaient jamais été lus. J’ai trouvé à la bibliothèque Mazarine à Paris (je n'ai pas noté dans ma liste précédente la bibliothèque Mazarine) des ouvrages qui n'avaient pas été coupés depuis le moment où ils étaient entrés, en 1604 par exemple! Çela fait un effet très douloureux. Quelquefois aussi on trouvait à la Bibliothèque Nationale, dans les années soixante, des livres qui avaient été offerts par leurs auteurs à la bibliothèque et qui étaient restés non coupés. On ne peut pas voir cela maintenant puisque les livres sont automatiquement coupés, mais autrefois on devait employer un coupe-papier pour ouvrir le livre. Vous avez des livres qui n'ont été coupés que pour les cinq, six premières pages. Ça m’angoisse un peu en tant qu'auteur... 


\section{Une grande partie des exemples que j'ai recensés et examinés, je les ai trouvés à la Bibliothèque Nationale,}

c'est normal, mais au moins autant à la bibliothèque de l'Arsenal. Et pourquoi cela? Parce que jusqu'à une date assez récente, la bibliothèque de l'Arsenal n'avait que de vieux catalogues manuscrits: des grands registres pour le fonds ancien. Et donc, il fallait aller y voir, car ils étaient, rangés par catégorie de livres. Et on avait là un ensemble important de livres de poésie du XVIe siècle. Aucun catalogue imprimé à la Bibliothèque Nationale ne recensait les livres du XVI ${ }^{e}$ siècle, ceux du XVII ${ }^{2}$.. je ne sais pas si cela existe maintenant. Les catalogues de la bibliothèque de l'Arsenal, étaient une aubaine pour qui recherchait, par exemple, les livres de poésie du XVIe siècle. J'ai observé que la plupart des anthologies de poèmes du XVI siècle qui ont été publiées ont été faites très probablement à partir du catalogue de l'Arsenal. Les auteurs ont trouvé les livres là. Et c'est très dommage de supprimer ces catalogues. C'est un des dangers de 
la numérisation, de l'informatisation, qui est la suppression des très vieux catalogues qui contiennent des merveilles. Ce n'est pas exactement le principe du bon voisin mais on trouva là des renseignements qui risquent d'être ensuite complètement perdus.

Au moment où je faisais cette étude, j'ai découvert, à ma grande surprise, qu'un nombre non négligeable de livres de sonnets, ou contenant au moins deux ou trois sonnets, se trouvait en exemplaire unique dans des bibliothèques le plus souvent municipales de province. À cette époque, pas d'internet, guère de possibilité technique pour aller trouver ce qu'il y avait dans ces bibliothèques. Vous écriviez, on ne vous répondait pas. Une seule solution: aller sur place. Ainsi j'ai rendu visite à un grand nombre de ces merveilleuses salles de lecture de villes grandes, moyennes, ou petites, où travaillaient silencieusement diverses personnes d'âge, de l'espèce qu'un historien anglais de la Révolution française a nommé les «érudits locaux». Je retourne dans mon souvenir et je vous en donne une liste; je ne pense pas qu'elle soit complète. Bien entendu, il y en a une très importante, c'est la bibliothèque de la Part-Dieu, la bibliothèque de 
Lyon. Mais il y a Versailles, Le Mans, Amiens, Caen, Besançon, Troyes, Montbrison (la bibliothèque de la Diana), Nancy, Grenoble, la bibliothèque de la faculté catholique de Lyon, Avignon, Aix, Blois, Dijon, Angers, la petite ville de Saintes, qui contient l'exemplaire unique des œuvres de Nicolas Arquesson (début du XVIIe), Carcassone, Nîmes, Poitiers, Arras, Bordeaux, Montpellier, Châlons-sur-Marne, Douai, Reims, Limoges, Carpentras. Dans toutes ces bibliothèques municipales, j'ai trouvé des livres qui ne sont pas à la Bibliothèque Nationale, et qui contiennent des poèmes du XVIe siècle ou du début du XVII .

Et voilà qu'au terme de ce parcours, finalement assez rapide, de mon expérience de lecteur en bibliothèque, je me rends compte de la chose suivante: à plusieurs reprises, ayant décidé d'un travail à faire, j'ai constaté qu'il m'obligeait à me rendre dans une ou plusieurs bibliothèques. J'avais déjà rendu visite à certaines, d'autres étaient nouvelles et s'ajoutaient alors à ce qu'on peut nommer une collection. C'est-à-dire que peu à peu, j'étais devenu un collectionneur de bibliothèques. Mais j'ai pris aussi conscience du fait qu'il me fallait alors considérer en fait que toutes ces recherches, 
que tous ces travaux auxquels je m'étais consacré si longtemps n'avaient eu qu'une cause unique. Ils étaient tout simplement des prétextes à satisfaire ce qui était devenu une passion, la bibliothécomanie. Je pensais que j'allais dans ces bibliothèques parce que je devais faire tel travail, mais pas du tout, j'avais choisi de faire tel travail parce que ça m'obligeait à aller dans des bibliothèques. Vous vous rendez compte!

Je terminerai aujourd'hui, sur une prévision, qui est de mon point de vue un peu pessimiste. Je ne suis pas totalement pessimiste mais ça dépend des jours. Je suis parfaitement sensible aux progrès que la technique donne, la possibilité de retenir pour moi une place à la bibliothèque de France sur mon bureau et de commander des livres. Depuis quelques temps, quand je commande (par exemple sur Amazon, où on peut commander des livres d'occasion publiés aux USA, et je lis beaucoup de littérature en anglais), je reçois des volumes ayant appartenu à des bibliothèques publiques de l'Arizona, du Kansas, de la Caroline du Nord etc. et qui ont été chassés de leurs rayons. Et je me dis que le temps n'est pas loin où les livres, sauf ceux qui, étant rares et précieux, auront Bipris le 
statut de pièces de musée, deviendront les parents pauvres des bibliothèques qui d'ailleurs, pour la plupart, comme cela se produit déjà de plus en plus, ne seront plus des bibliothèques mais des médiathèques et que le lieu où je suis venu aujourd'hui vous parler, dans quelques temps ne sera plus nommé enssib mais enssim. Heureusement, je ne serai plus là. 
Ceci n'est pas une biographie; une tentative infra ordinaire; une promenade. Car Jacques Roubaud marche; surtout au Palais Royal, le matin; en pataugas de toile noire, beige, ou verte, c'est selon. Marcher dans Paris, sans but véritable, sans obligation, est une occasion de poésie, écrit-il: «la poésie, selon mon expérience, nait de la marche, principalement». Jacques Roubaud est d'abord un mathématicien, fasciné par le groupe Bourbaki et Gustave Choquet; Jacques Roubaud est poète; il se revendique comme tel; spécialiste de la sextine et du sonnet; il est membre de l'Oulipo, coopté en 1966 par Raymond Queneau; inventeur de la contrainte du baobab; collectionneur de bibliothèques. Jacques Roubaud, né en 1932, à Caluire et Cuire, Rhône, dix ans, six mois et seize jours avant que Jean Moulin n'y soit arrêté, deux formes de résistance, l'un, les armes à la main, l'autre avec le langage, est un fidèle compagnon du Théâtre National Populaire de Villeurbanne. Voici donc notre homme, mathématicien, poète, traducteur, homme de théâtre, sortant des jardins du palais Royal. Il rentre chez lui. Nous l'accompagnons en pensée. Ce n'était donc qu'une déambulation de 1200 signes. 


\section{Quelques œuvres citées}

Ferdinand Buisson, Sébastien Castellion, sa vie, son œuvre, Hachette, Paris, 1892

< http://gallica.bnf.fr/ark:/12148/bpt6k209976b.r=castellion.langFR >

Raymond Queneau, Pierrot mon ami, Gallimard, 1942

Paul Bénichou, Romantismes français, Gallimard, 2004

Frédéric Boyer (dir.), La bible, Bayard, 2009

Les autres ouvrages édités à l'occasion des 20 ans de l'enssib

Imaginaires des bibliothèques: "Mondes lettrés:

fragments d'un abécédaire" par Christian Jacob, «< http://www.fonds-thorez.ivry94.fr/thorez >» par Annette Wieviorka.

[Parution juin 2012 - ISBN 978-2-910227-96-8]

Tours et détours en bibliothèque: Carnet de voyage 1992-2012, ouvrage collectif réunissant dix-neuf écrivains, une photographe et vingt bibliothèques. [Parution octobre 2012 - ISBN 978-2-910227-97-5]

Architecture des bibliothèques: 1992-2012, ouvrage collectif réunissant des architectes, des conservateurs de bibliothèques, des élus et plus de vingt bibliothèques. L'illustration de l'ouvrage est confiée à Frank Bonnefoy. [Parution novembre 2012 - ISBN 978-2-910227-98-2] 
Lire, écrire ou comment je suis devenu collectionneur de bibliothèques de Jacques Roubaud a été achevé d'imprimer le 25 mai 2012 à l'imprimerie Chirat à Saint-Just-la-Pendue (42540) pour le compte des Presses de l'enssib, à l'occasion des 20 ans de l'école.

Une version numérique de cet ouvrage est disponible gratuitement: < http://roubaudlireecrire.enssib.fr >

II a été édité un tirage de tête de 350 exemplaires, hors commerce.

Couverture: Jacques Roubaud, 23 janvier 2008, conférence filmée, enssib, disponible sur : <http://www.enssib.fr/bibliotheque-numerique/document-1709 >

Conception graphique et mise en page:

atelier Perluette, Florence Roller

$<$ http://perluette-atelier-com >

Coordination éditoriale :

Thierry Ermakoff, Catherine Jackson, enssib

Transcription de la conférence: Carole Laborié, enssib

Relecture: Célestino Avelar, enssib

Cet ouvrage a été composé en caractères

Caecilia et Turlot.

Dépôt légal: deuxième trimestre 2012 
Lire, écrire ou comment je suis devenu collectionneur de bibliothèques de Jacques Roubaud a été achevé d'imprimer le 25 mai 2012 à l'imprimerie Chirat à Saint-Just-la-Pendue (42540) pour le compte des Presses de l'enssib, à l'occasion des 20 ans de l'école.

Une version numérique de cet ouvrage est disponible gratuitement: < http://roubaudlireecrire.enssib.fr >

II a été édité un tirage de tête de 350 exemplaires, hors commerce.

Couverture: Jacques Roubaud, 23 janvier 2008, conférence filmée, enssib, disponible sur : <http://www.enssib.fr/bibliotheque-numerique/document-1709 >

Conception graphique et mise en page:

atelier Perluette, Florence Roller

$<$ http://perluette-atelier-com >

Coordination éditoriale :

Thierry Ermakoff, Catherine Jackson, enssib

Transcription de la conférence: Carole Laborié, enssib

Relecture: Célestino Avelar, enssib

Cet ouvrage a été composé en caractères

Caecilia et Turlot.

Dépôt légal: deuxième trimestre 2012 

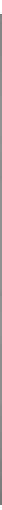

\section{enu collectionneur de bibliothèques}

Jacques Roubaud, poète, mathématicien, mathématicien et poète, homme de théâtre, traducteur, membre de l'oulipo, est aussi et d'abord un collectionneur de bibliothèques: les plus prestigieuses (la British Library, la Bibliothèque nationale, square Louvois, la Bibliothèque nationale de France, quai de Tolbiac, la bibliothèque Sainte-Geneviève), les plus rares (l'Inguimbertine), et les plus improbables sont entrées dans son catalogue; imprimé; c'est à sa visite qu'il nous invite. 